\title{
A systematic review of the effectiveness of qigong exercise in supportive cancer care
}

\author{
Cecilia L. W. Chan - Chong-Wen Wang • \\ Rainbow T. H. Ho • Siu-Man Ng • Jessie S. M. Chan • \\ Eric T. C. Ziea • Vivian C. W. Wong
}

Received: 1 February 2011 / Accepted: 29 December 2011 / Published online: 19 January 2012

(C) The Author(s) 2012. This article is published with open access at Springerlink.com

\begin{abstract}
Purpose Qigong as a complementary and alternative modality of traditional Chinese medicine is often used by cancer patients to manage their symptoms. The aim of this systematic review is to critically evaluate the effectiveness of qigong exercise in cancer care.

Methods Thirteen databases were searched from their inceptions through November 2010. All controlled clinical trials of qigong exercise among cancer patients were included. The strength of the evidence was evaluated for all included studies using the Oxford Centre for Evidence-based Medicine Levels of Evidence. The validity of randomized controlled trials (RCTs) was also evaluated using the Jadad Scale.

Results Twenty-three studies including eight RCTs and fifteen non-randomized controlled clinical trials (CCTs) were identified. The effects of qigong on physical and psychosocial outcomes were examined in 14 studies and the effects on biomedical outcomes were examined in 15 studies. For physical and psychosocial outcomes, it is difficult to draw a conclusion due to heterogeneity of outcome measures and variability of the results in the included studies. Among reviewed studies on biomedical outcomes, a consistent tendency appears to emerge which suggests that the patients
\end{abstract}

C. L. W. Chan • C.-W. Wang $(\bowtie) \cdot$ R. T. H. Ho · S.-M. Ng • J. S. M. Chan

Centre on Behavioral Health, The University of Hong Kong,

HKSAR, China,

5 Sassoon Road,

Pokfulam, Hong Kong, China

e-mail: wangcw@hku.hk

E. T. C. Ziea $\cdot$ V. C. W. Wong

Chinese Medicine Department, Hospital Authority, HKSAR, Hong Kong, China treated with qigong exercise in combination with conventional methods had significant improvement in immune function than the patients treated with conventional methods alone.

Conclusions Due to high risk of bias and methodological problems in the majority of included studies, it is still too early to draw conclusive statements. Further vigorously designed large-scale RCTs with validated outcome measures are needed.

\section{Keywords Cancer $\cdot$ Qigong $\cdot$ Exercise $\cdot \mathrm{RCT} \cdot \mathrm{CAM}$}

\section{Introduction}

As a major chronic illness and one of the leading causes of mortality, cancer poses adverse and debilitating impacts on the patients' functional capacity and quality of life. Cancer patients often experience painful symptoms and side effects following treatments such as surgery, chemotherapy, and radiotherapy. An increasing use of complementary and alternative medicine (CAM) by cancer patients as a supplement to the mainstream Western medical interventions has been evident in the literature [1-3]. Patients with cancer usually employ CAM for promoting health and managing symptoms experienced during active treatment and the period of survivorship [4].

Qigong, a general term for a large range of traditional Chinese energy exercises and therapies, is a form of traditional Chinese medicine (TCM) practice. It is popularly practiced by a large number of people in Chinese communities. Basically, there are two categories of qigong: internal qigong versus external qigong. Internal qigong or qigong exercise is self directed and involves the use of movements, 
meditation, and control of breathing pattern. The gentle movements and postures of the exercise are designed to achieve a harmonious flow of energy $(q i)$ in the body so as to improve physical fitness and overall well-being. External qigong is usually performed by a trained practitioner using their hands to direct emitted "qi" energy onto the patient's body in diagnosis and treatment of various diseases. RCTs have shown efficacy for some TCM therapies [5], but the efficacy of other complementary and alternative modalities, particularly qigong, remains uncertain despite its popularity among chronic patients. One earlier review of qigong for cancer treatment did not differentiate between internal and external qigong [6, 7]. However, the mechanism of its effect is different for internal and external qigong, so their efficacy may not be the same. One recent review of qigong exercise for cancer treatment did not examine the efficacy of qigong exercise on different health outcomes critically and did not consider biomedical outcomes $[8,9]$. Thus, the primary objective of this review is to examine the overall efficacy and effectiveness of qigong exercise in cancer care, with a particular focus on different health outcomes.

\section{Methods}

The following electronic databases were searched from their respective inceptions through November 2010: The Cochrane Central Register of Controlled Trials (CENTRAL), PubMed/MEDLINE, The Cumulative Index to Nursing and Allied Health Literature (CINAHL), Excerpta Medica Database (Embase), Allied and Complementary Medicine Database (AMED), Qigong and Energy Medicine Database, China Journals Full-text Database-Medicine/ Hygiene Series, China Proceedings of Conference Full-text Database, Chinese Master Theses Full-text Database, China Doctor Dissertations Full-text Database, Electronic Theses and Dissertation System (Taiwan), Taiwan Electronic Periodical Services, and Index to Taiwan Periodical Literature System. The search terms used for this review included: qigong, qi-gong, qi gong, chi chung, chi gong, qi chung, cancer, tumor, carcinoma, and neoplasm. Both traditional and simplified Chinese translations of these terms were used in Chinese databases. Reference lists of all included studies, existing reviews, and other archives of the located publications were hand searched for further relevant studies.

All controlled clinical trials among cancer patients who received qigong intervention alone or combined with other treatments, either on an individual basis or as group sessions, were included. RCTs were preferred and CCTs including cohort studies and case-control studies were also included to provide alternative evidence due to limited number of RCTs in the field. Uncontrolled observational studies were excluded. Due to the focus on internal qigong in this systematic review, studies on external qigong were excluded. For all included studies, the primary data from the original sources were reviewed and analyzed.

All available evidence of the health benefits of qigong exercise for cancer patients was considered. Specifically, the outcome measures for this review included indicators of physical health such as symptoms, measures of psychosocial health such as quality of life, and biomedical outcomes. For each included study, data were extracted by one main researcher and then verified by another researcher. Any discrepancies were resolved by discussion. The strength of the evidence was evaluated for all the included studies using the Oxford Centre for Evidence-based Medicine Levels of Evidence [10]. These criteria grade the methodological rigor of studies from level 1 or grade A (systematic review of RCTs, 1a; individual RCT with narrow confidence interval, 1b) to level 5 or grade $\mathrm{D}$ (expert opinion). The quality and validity of the included RCTs were also evaluated using the Jadad Scale [11], which is based on three criteria: description of randomization, blinding, and patient attrition (the score ranges from 0 to 5 , the higher the better). This scale has been continually used in systematic reviews.

Internal validity for all included controlled trials, including selection bias, performance bias, detection bias, and attrition bias, were assessed using the framework for methodological quality recommended by Jüni et al. [12]. Risk of bias for the included RCTs was also evaluated on the basis of the quality of their randomization procedure and allocation concealment, blinding, and incomplete outcome data assessment, as recommended in the Cochrane Handbook [13].

\section{Results}

Our search returned 261 relevant references. Any studies that were (1) not related to qigong or cancer, (2) not a clinical trial, (3) not a controlled study, (4) studies on external qigong or emitted "qi", (5) studies with unparallel control design, (6) case reports, or (7) duplicative publications were excluded. Consequently, 23 studies including eight RCTs [14-21] and fifteen CCTs [22-36], published between 1988 and 2010, were identified for inclusion in this review. Of them, 14 studies examined the effects of qigong exercise on physical and psychosocial outcomes and 15 studies examined the effects on biomedical outcomes (including six studies on both). Characteristics of the included studies on physical and psychosocial outcomes are presented in Table 1, and the studies on biomedical outcomes are presented in Table 2.

The samples in the included studies ranged from 20 to 229 . A two-armed, parallel group design was used in nearly all of the included studies except two RCTs. One RCT [21] was conducted with a three-armed parallel group design and 


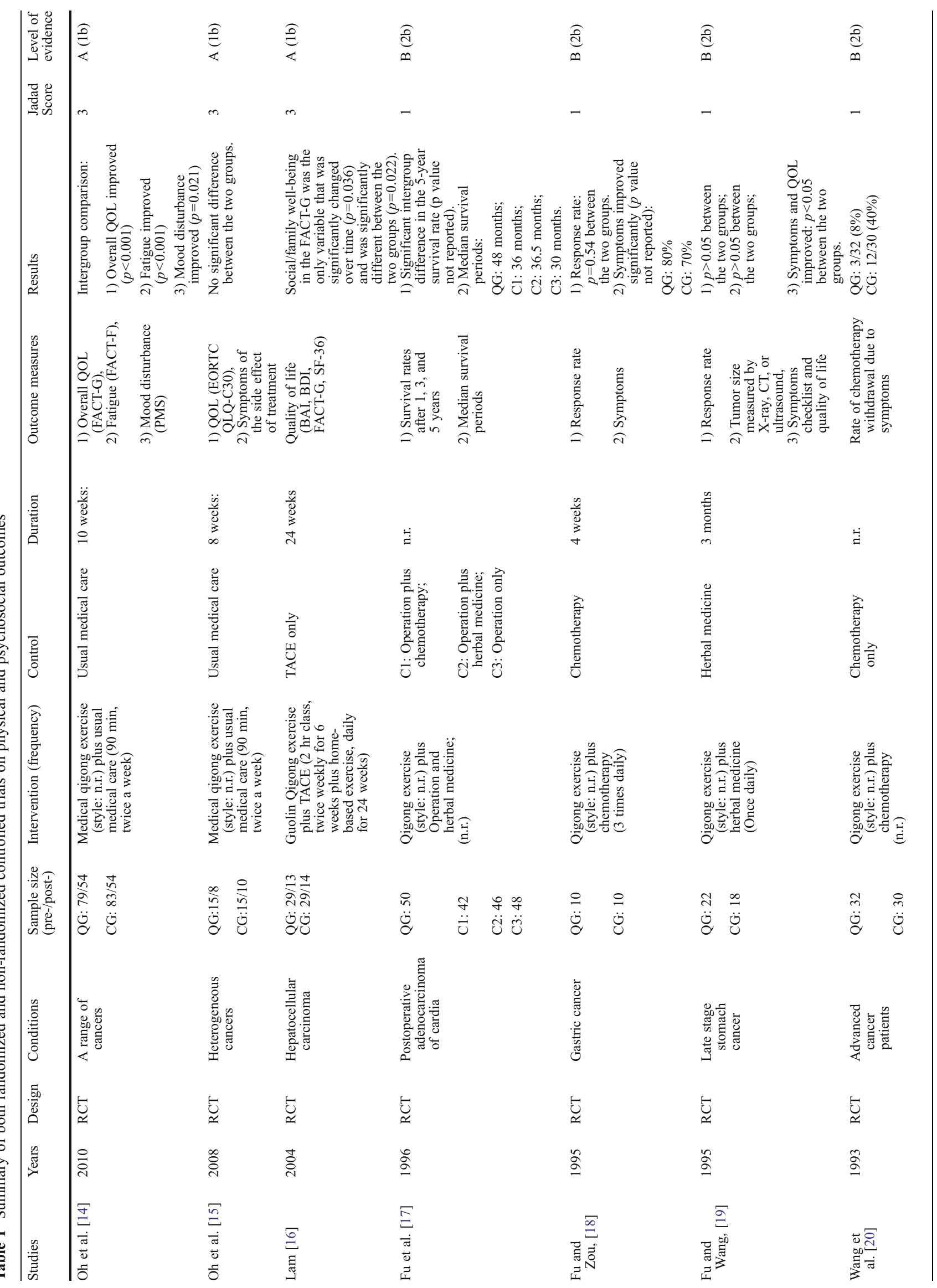




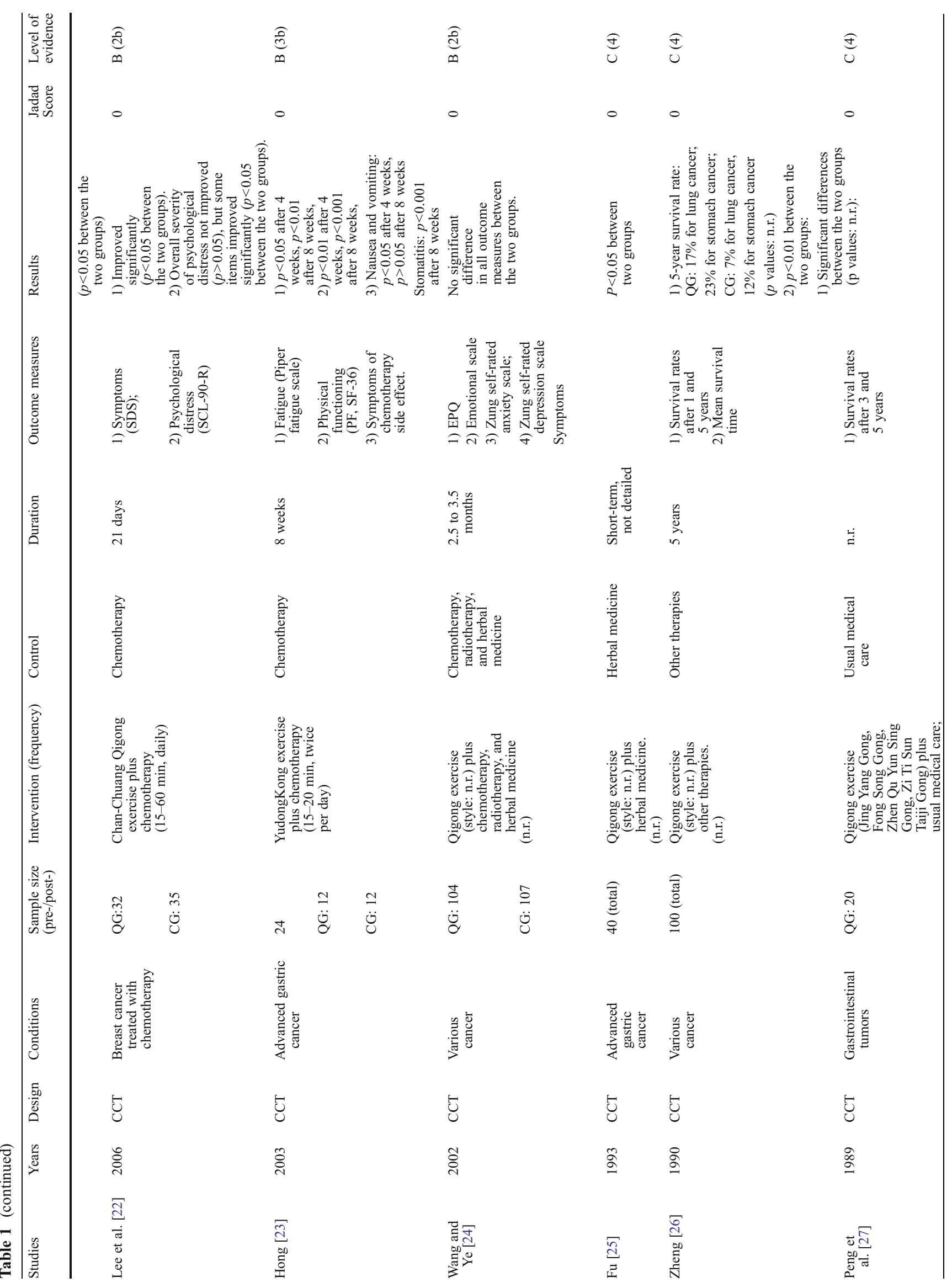




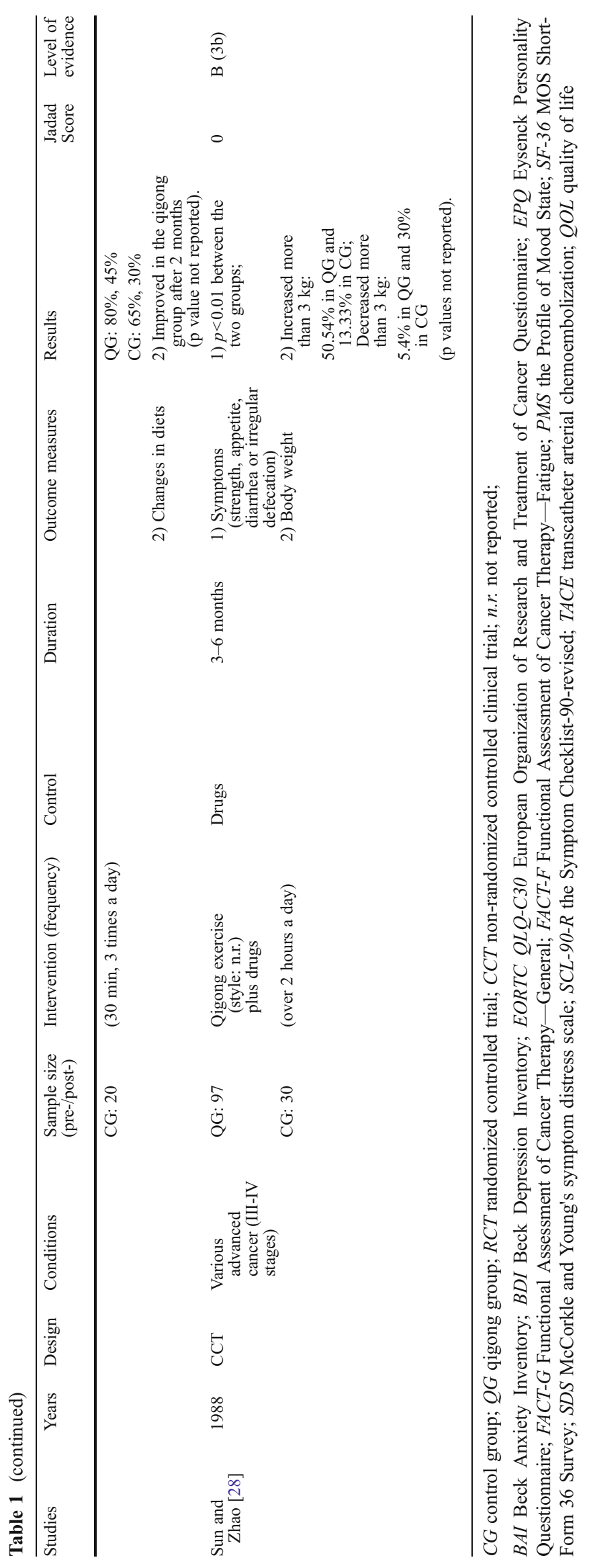




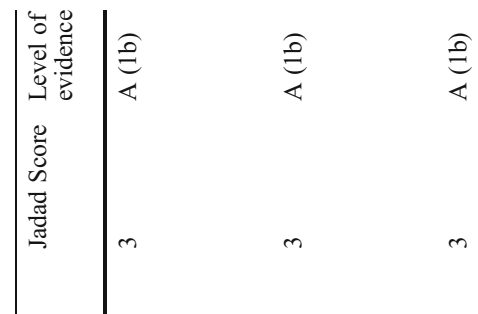

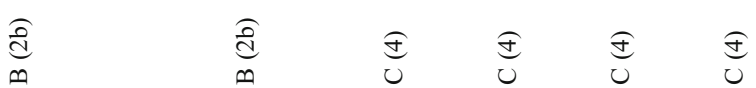
$\underset{Ð}{Ð}$

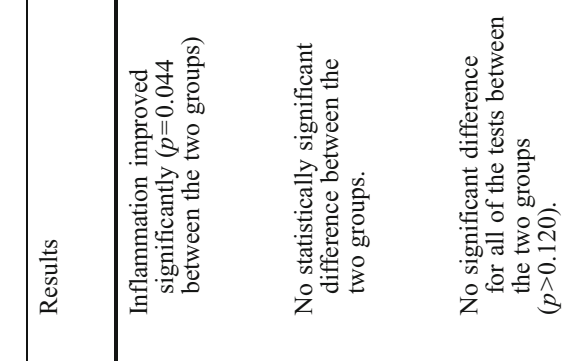

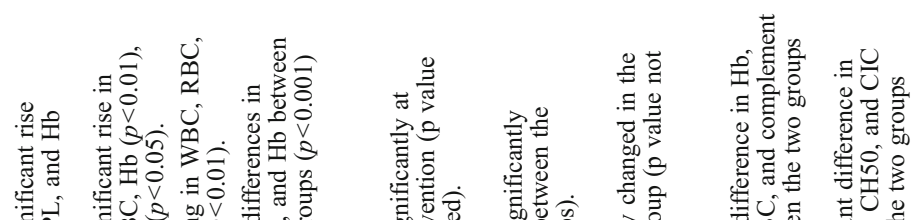 $\circ$

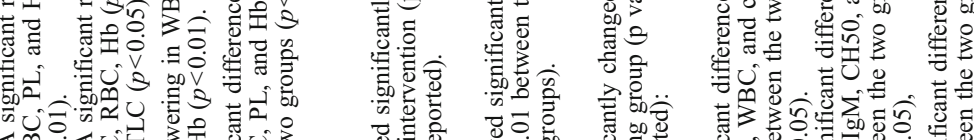

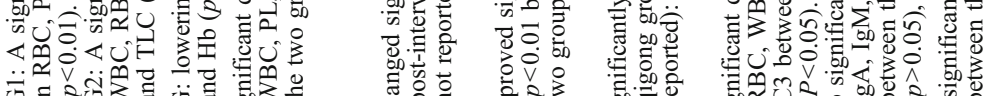

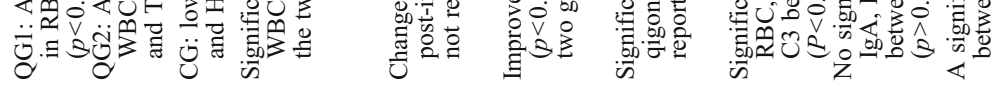

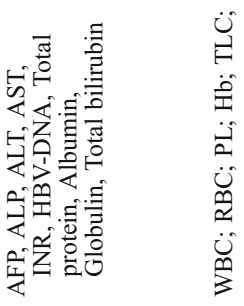

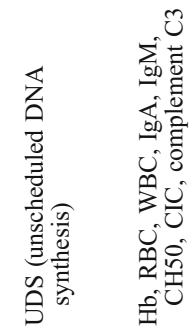

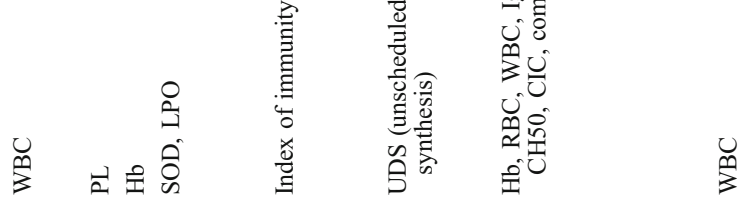
$\frac{5}{5}$
童

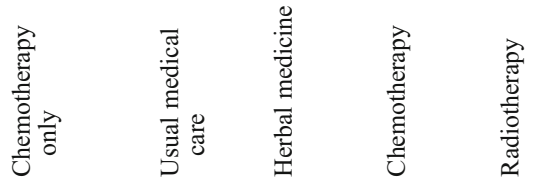

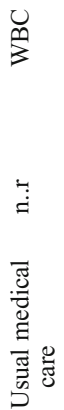

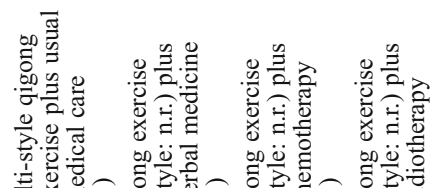

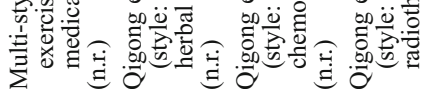

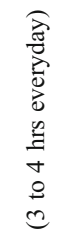

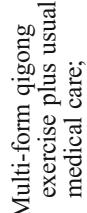

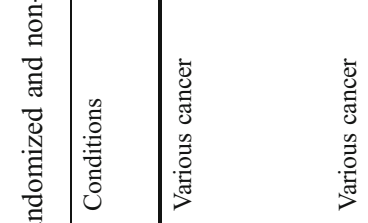

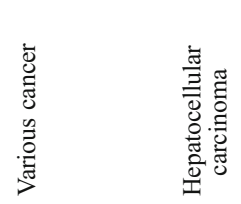

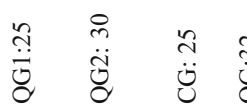
光

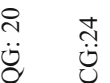

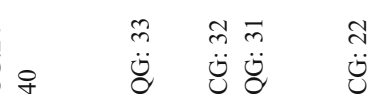

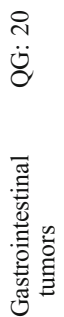

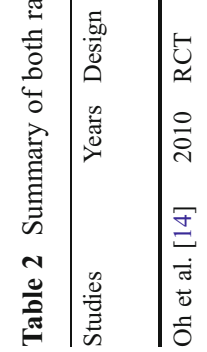

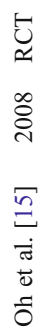

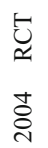
$\xi$

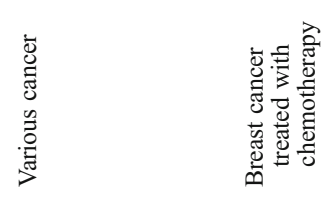

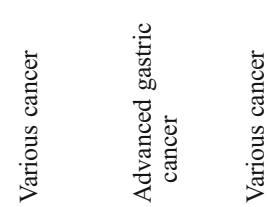

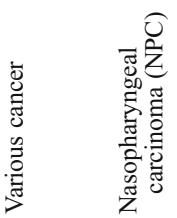

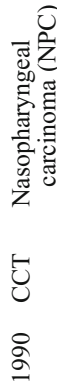
$\stackrel{\infty}{\infty}$
包
E
总

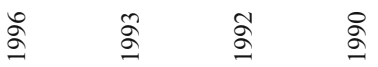

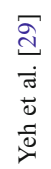

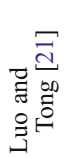

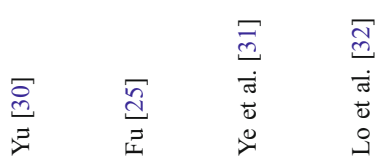

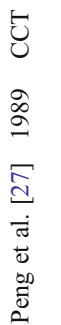




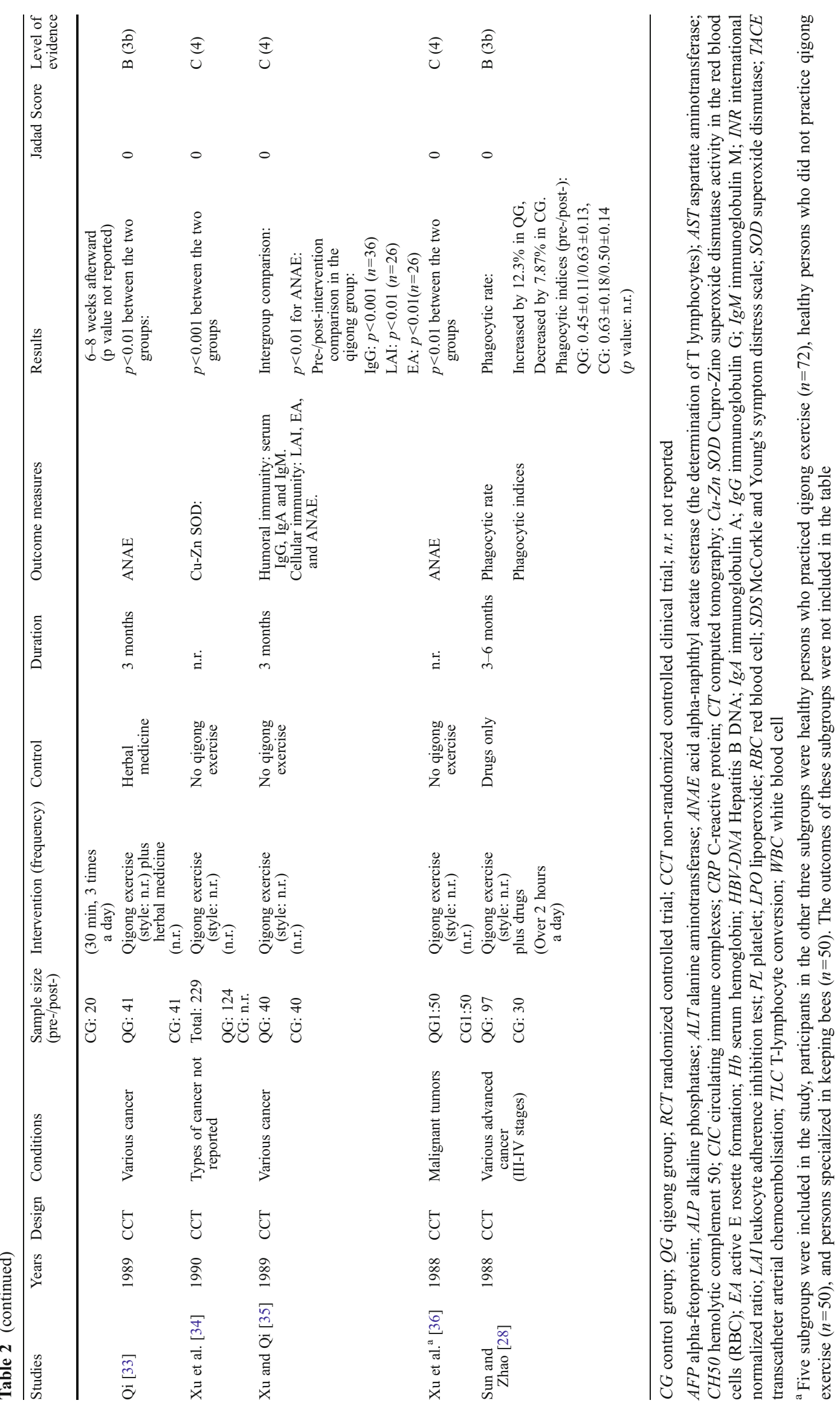


another RCT [17] with a four-armed parallel group design. The number of participants in each subgroup was reported in most of the included studies, except two studies $[25,26]$ in which only the number of total sample was reported. We assumed that participants in the two studies were equally allocated to each subgroup. Thus, the included studies on physical and psychosocial outcomes covered 572 cancer patients in qigong groups (ranging from 10 to 104) and 595 cancer patients in control groups (ranging from 10 to 136), and the studies on biomedical outcomes covered 656 cancer patients in qigong groups (ranging from 15 to 124) and 601 cancer patients in control groups (ranging from 15 to 105). Of the 23 included studies, ten studies focused on single type of cancer such as gastric cancer [18, 19, 23, 25, 27], adenocarcinoma of cardia [17], hepatocellular carcinoma [16], breast cancer [22, 29], and nasopharyngeal carcinoma [32]; others employed a sample including multiple types of cancer.

Interventions employed in these studies included qigong plus herbal medicine [19, 25, 33] or drugs [28], chemotherapy [16, 18, 20, 22, 23, 29, 31], radiotherapy [32], usual medical care $[14,15,27,30]$, or multiple therapies [24]. In a study with three-armed parallel group design [21], the intervention group with qigong exercise plus chemotherapy was compared with two control groups (qigong exercise alone and chemotherapy alone). In another study with a fourarmed parallel group design [17], the intervention group with qigong exercise plus surgery and herbal medicine was compared with three control groups (surgery plus chemotherapy, surgery plus herbal medicine, and surgery only). Durations of qigong intervention ranged from 21 days to 5 years, with no longer than 2 months for six studies $[15,18$, 21-23, 29], from 10 weeks to 6 months for nine studies [14, $16,19,24,28,30,31,33,35]$, and as long as 5 years for three studies $[17,26,27]$. Duration of intervention was not reported in five studies [20, 25, 32, 34, 35].

The emerged physical and psychosocial outcomes (Table 3) in these studies included self-perceived symptoms [15, 18-20, $22,23,25,28]$, quality of life (QOL) [14-16, 19], psychological distress [14, 16, 22, 24], survival rate [16, 17, 26], response rate $[18,19]$, fatigue $[14,23]$, physical functioning [23], body weight [28], and tumor size [19]. Biomedical outcomes (Table 4) that emerged in these studies included "inflammatory markers" (C-reactive protein) [14, 15], parameters of immunity such as immunoglobulin and complements [25, 28, 32-35], the numbers or the proportions of blood cells or their antioxidize capacity [21, 27, 29-32, 34], and hepatic function [16].

\section{Effects of interventions}

Four RCTs [14-16, 19] examined the effect of qigong exercise on QOL. Two studies showed that overall QOL was improved significantly in the qigong group compared with the control group $[14,19]$. In two other studies, QOL was not improved significantly in the qigong group compared with controls $[15,16]$. Four studies including two RCTs [14, 16], and two CCTs [22, 24] evaluated the effects of qigong exercise on psychological distress [22], mood disturbance [14], anxiety, and depression [16, 24]. Only one study [14] revealed that mood status was improved significantly when compared with the control group receiving usual medical care $(p<0.05)$.

Eight studies including three RCTs $[15,18,20]$ and five CCTs $[19,22,23,25,28]$ assessed the effect of qigong exercise on the improvement of symptoms among cancer patients. All of the five CCTs generated a significant result. Of the three RCTs, only one [19] indicated that fewer participants in the qigong group suspended chemotherapy due to symptoms compared to a control group ( $8 \%$ vs. $40 \%$, $p<0.05)$. Results of two other RCTs showed that the difference for symptom improvement between the qigong group and the control group was not statistically significant.

Two studies including one RCT [14] and one CCT [23] examined the efficacy of qigong exercise in improving fatigue among patients with various cancers. Results of both studies indicated that fatigue in the qigong group was significantly improved at post-intervention compared with the control group. One of them [23] also examined the effect of qigong exercise on physical functioning, and the results demonstrated a significant difference between the two groups in the level of difficulty with daily activities at post-intervention after controlling for baseline variables.

Two RCTs examining the effect of qigong combined with chemotherapy [18] or herbal medicine [19] on short-term "response rate" among patients with late-stage gastric cancer did not show a statistically significant difference between the qigong group and the control group. However, three studies including one RCT [17] and two CCTs [26, 27] on long-term survival rate among cancer patients reported a significant difference in the 5-year survival rate between those practicing qigong and controls.

One RCT [19] examined the effect of qigong exercise plus herbal medicine on tumor size in 40 elderly patients with latestage stomach cancer, who were either too old or too far along in their diagnosis to undergo surgery. Tumor size was measured with objective measures such as X-ray, CT scan, biopsy, and/or ultra-sound. No significant difference was found between the qigong group and a control group after 3 months of intensive treatment. One CCT [28] evaluated the effect of qigong exercise on the change of body weight among cancer patients. At the end of the 3-month trial, body weight increased more than $3 \mathrm{~kg}$ in half $(50.5 \%)$ of participants in the qigong group and one-eighth (13.3\%) in the control group; meanwhile, $5.4 \%$ in the qigong group and $30 \%$ in the control group lost more than $3 \mathrm{~kg}$ in body weight.

Two RCTs by the same research team $[14,15]$ examined the effect of qigong exercise on a primary biomarker of inflammation 
Table 3 Distribution of studies across physical and psychosocial outcomes

\begin{tabular}{|c|c|c|c|c|c|}
\hline Outcomes & Studies & Design & $n$ (QG group) & Duration & Results \\
\hline \multirow[t]{4}{*}{ Quality of life (QOL): } & Oh et al. [14] & RCT & 54 & 10 weeks & $p<0.001$ \\
\hline & Oh et al. [15] & $\mathrm{RCT}$ & 15 & 5 weeks & n.s. \\
\hline & Lam [16] & $\mathrm{RCT}$ & 13 & 24 weeks & n.s. \\
\hline & Fu and Wang [19] & $\mathrm{RCT}$ & 22 & 3 months & $p<0.05$ \\
\hline \multirow[t]{8}{*}{ Symptoms } & Oh et al. [15] & $\mathrm{RCT}$ & 15 & 5 weeks & n.s. \\
\hline & Fu and Zou [18] & $\mathrm{RCT}$ & 10 & 4 weeks & n.s. \\
\hline & Lee et al. [22] & $\mathrm{CCT}$ & 32 & 21 days & $p<0.05$ \\
\hline & Hong [23] & $\mathrm{CCT}$ & $24 / 2$ & 8 weeks & $\begin{array}{l}p<0.05 \text { after } 4 \text { weeks } \\
p>0.05 \text { after } 8 \text { weeks }\end{array}$ \\
\hline & Fu and Wang [19] & $\mathrm{RCT}$ & 22 & 3 months & $p<0.05$ \\
\hline & $\mathrm{Fu}[25]$ & $\mathrm{CCT}$ & 22 & 3 months & $p<0.05$ \\
\hline & Wang et al. [20] & $\mathrm{RCT}$ & 32 & - & $p<0.05$ \\
\hline & Sun and Zhao [28] & $\mathrm{CCT}$ & 97 & 3 months & $p<0.01$ \\
\hline \multirow[t]{4}{*}{ Psychological well-being } & $\begin{array}{l}\text { Oh et al. [14] } \\
\text { (mood disturbance) }\end{array}$ & RCT & 54 & 10 weeks & $p<0.05$ \\
\hline & $\begin{array}{l}\text { Lee, et al. [22] } \\
\text { (psychological distress) }\end{array}$ & $\mathrm{CCT}$ & 32 & 21 days & n s. \\
\hline & $\begin{array}{l}\text { Wang and Ye [24] } \\
\text { (anxiety and depression) }\end{array}$ & $\mathrm{CCT}$ & 104 & $2.5-3.5$ months & n.s. \\
\hline & $\begin{array}{l}\text { Lam [16] } \\
\text { (anxiety and depression) }\end{array}$ & RCT & 13 & 24 weeks & n.s. \\
\hline Physical functioning & Hong [23] & $\mathrm{CCT}$ & $24 / 2$ & 8 weeks & $p<0.01$ \\
\hline \multirow[t]{2}{*}{ Fatigue } & Oh et al. [14] & $\mathrm{RCT}$ & 54 & 10 weeks & $p<0.01$ \\
\hline & Hong [23] & $\mathrm{CCT}$ & $24 / 2$ & 8 weeks & $p<0.01$ \\
\hline \multirow[t]{3}{*}{ Survival rate } & Fu et al. [17] & $\mathrm{RCT}$ & 50 & 5 years & $p<0.05$ \\
\hline & Peng et al. [27] & $\mathrm{CCT}$ & 20 & 5 years & $p<0.05$ \\
\hline & Zheng [26] & $\mathrm{CCT}$ & $100 / 2$ & 5 years & $p<0.01$ \\
\hline \multirow[t]{2}{*}{ Response rate } & $\mathrm{Fu}$ and $\mathrm{Zou}[18]$ & RCT & 10 & 4 weeks & n.s. \\
\hline & Fu and Wang [19] & $\mathrm{RCT}$ & 22 & 3 months & n.s. \\
\hline Tumor size & Fu and Wang [19] & RCT & 22 & 3 months & n.s. \\
\hline Body weight & Sun and Zhao [28] & $\mathrm{CCT}$ & 97 & 3 months & $p<0.05$ \\
\hline
\end{tabular}

$Q G$ qigong; $R C T$ randomized controlled trial; $C C T$ non-randomized controlled trial; n.s. no significance

- serum C-reactive protein (CRP), which has been found in several studies to be an independent predictor of cancer prognosis. In a pilot study [15], the results indicated no significant difference in the level of CRP between the qigong group and a control group at post-intervention. In a subsequent large-scale study [14], the results indicated that inflammation was significantly improved in the qigong group at the end of 10 weeks intervention, compared with the control group with usual care after controlling for baseline variables $(p<0.05)$.

Seven studies $[25,28,31-33,35,36]$ investigated the impacts of qigong exercise for immune function with a great variety of outcome parameters including phagocyte rate [28], acid alpha-naphthyl acetate esterase (ANAE) - the determination of T lymphocytes [33, 35, 36], immunoglobulin A (IgA), and immunoglobulin $\mathrm{M}(\operatorname{IgM})[32,35]$, complement 3 [32], and the rate of unscheduled DNA synthesis (UDS) in peripheral blood lymphocytes [31]. Overall, the results of these studies demonstrated a favorable effect of qigong exercise on immune function, except for two studies with no significant improvement in the levels of $\operatorname{IgA}$ and $\operatorname{IgM}[32,35]$.

Four studies [21, 27, 29, 32] assessed the effects of qigong exercise on the numbers or the levels of components in peripheral blood, and two additional studies [30, 34] examined the anti-oxidize capacity of blood components. All of these studies revealed a desirable result. One study [16] testing the effect of qigong combined with transcatheter arterial chemoembolization (TACE) on hepatic function in patients with hepatocellular carcinoma indicated that no significant improvement in hepatic function was found after 24 weeks intervention. 
Table 4 Distribution of studies across biomedical outcomes

\begin{tabular}{|c|c|c|c|c|c|c|}
\hline Outcomes & Studies & Design & Biomedical outcomes & $n$ (QG group) & Duration & Results \\
\hline \multirow[t]{2}{*}{ Inflammatory marker } & Oh et al. [14] & RCT & Serum CRP & 54 & 10 weeks & $p>0.05$ \\
\hline & Oh et al. [15] & $\mathrm{RCT}$ & Serum CRP & 15 & 5 weeks & $p<0.05$ \\
\hline \multirow[t]{11}{*}{ Immune function } & Sun and Zhao [28] & $\mathrm{CCT}$ & Phagocytic rate & 97 & 3 months & $p<0.05$ \\
\hline & $\mathrm{Fu}[25]$ & $\mathrm{CCT}$ & "Index of immunity" & $40 / 2$ & n.r. & $p<0.01$ \\
\hline & $\mathrm{Xu}$ et al. [36] & $\mathrm{CCT}$ & ANAE & 50 & n.r. & $p<0.01$ \\
\hline & Qi [33] & $\mathrm{CCT}$ & ANAE & 41 & 3 months & $p<0.01$ \\
\hline & $\mathrm{Xu}$ and Qi [35] & $\mathrm{CCT}$ & ANAE & 40 & 3 months & $p<0.01$ \\
\hline & & & $\operatorname{IgA}, \operatorname{IgM}$ & & & $p>0.05$ \\
\hline & & & InG, LAI, EA & & & $p<0.01$ \\
\hline & & & & & & (pre- vs. post-) \\
\hline & Lo et al. [32] & $\mathrm{CCT}$ & $\mathrm{C} 3$ & 31 & n.r. & $p<0.05$ \\
\hline & & & IgA, IgM, Ch50, CIC & & & $p>0.05$ \\
\hline & Ye et al. [31] & $\mathrm{CCT}$ & UDS & 33 & 3 months & $p<0.05$ \\
\hline \multirow{6}{*}{$\begin{array}{l}\text { Blood cells and } \\
\text { anti-oxidize } \\
\text { capacity }\end{array}$} & Yeh et al. [29] & $\mathrm{CCT}$ & WBC, RBC, $\mathrm{Hb}$ & 32 & 21 days & $p<0.01$ \\
\hline & Luo and Tong [21] & RCT & WBC, RBC, $\mathrm{Hb}$ & 25 & 60 days & $p<0.05$ \\
\hline & Peng et al. [27] & $\mathrm{CCT}$ & WBC, RBC, $\mathrm{Hb}$ & 20 & 5 years & $p<0.05$ \\
\hline & Lo et al. [32] & $\mathrm{CCT}$ & WBC, RBC, $\mathrm{Hb}$ & 31 & n.r. & $p<0.05$ \\
\hline & Xu et al. [34] & $\mathrm{CCT}$ & $\mathrm{Cu}-\mathrm{Zn} \mathrm{SOD}$ & 124 & n.r. & $p<0.01$ \\
\hline & $\mathrm{Yu}[30]$ & $\mathrm{CCT}$ & SOD, LPO & 20 & 6 months & $p<0.01$ \\
\hline Hepatic function & Lam [16] & $\mathrm{RCT}$ & $\begin{array}{l}\text { AFP, ALP, ALT, AST, albumin, } \\
\text { globulin, total protein }\end{array}$ & 13 & 24 weeks & n. s. \\
\hline
\end{tabular}

$Q G$ qigong; $R C T$ randomized controlled trial; $C C T$ non-randomized controlled clinical trial

n.r. not reported; n.s. no significance

AFP Alpha-fetoprotein; $A L P$ alkaline phosphatase; $A L T$ alanine aminotransferase; $A N A E$ acid alpha- naphthyl acetate esterase (the determination of T lymphocytes); $A S T$ aspartate aminotransferase; $C H 50$ hemolytic complement 50; $C 3$ complement 3; $C I C$ circulating immune complexes; $C R P$ Creactive protein; $C T$ computed tomography; $\mathrm{Cu}$-Zn SOD cupro-zino superoxide dismutase activity in the red blood cells (RBC); $E A$ active E rosette formation; $H b$ serum hemoglobin; $H B V-D N A$ hepatitis B DNA; $I g A$ immunoglobulin A; $I g G$ immunoglobulin $\mathrm{G}$; IgM immunoglobulin M; INR international normalized ratio; $L A I$ leukocyte adherence inhibition test; $P L$ platelet; $L P O$ lipoperoxide; $R B C$ red blood cell; $S D S$ McCorkle and Young's symptom distress scale; $S O D$ superoxide dismutase; TACE transcatheter arterial chemoembolization; TLC T-lymphocyte conversion; WBC white blood cell

\section{Risk of bias in included studies}

Of the 23 included studies, only 8 are RCTs, and the 15 included CCTs may have a high risk of selection bias and the strength of evidence for the results of these studies is limited due to their non-randomized design. Of the eight included RCTs, only three studies [14-16] described the method of randomization, one study [16] described details about allocation concealment, and three studies [14-16] reported details of drop-outs and withdrawals that might lead to exclusion of some particular patients. Other studies did not describe their methods of sequence generation or allocation concealment and drop-outs and were rated as "unclear" for those domains within this review. Given the nature of the qigong exercise intervention, blinding of participants and intervention delivery was generally infeasible. However, none of the included studies made an attempt to blind the assessors. Thus, a risk for biases might be introduced in most of the included RCTs.
These studies were conducted in Australia [14, 15], Korea [23], Taiwan [22, 29], Hong Kong [16], and mainland China [17-20, 24-28, 30-36]. Only four of them were published in peer-review journals in English [14, 15, 22, 29], and two were published in Chinese academic journals [24, 33]. Two studies were unpublished theses [16, 23], and two other studies were published in a book $[18,19]$. The remaining thirteen studies were presented in conferences on medical qigong without detailed information provided [17, 20, 21, 25-28, 30-32, 34-36]. These studies had not undergone the process of peer review, thus introducing to the potential for a number of biases.

\section{Discussion}

In this systematic review, the effectiveness of qigong exercise on multiple health outcomes in supportive care to cancer patients was examined comprehensively. To summarize, 23 controlled trials including 8 RCTs and 15 CCTs 
were included in this review. Five RCTs suggested favorable effects of qigong exercise on the improvement of symptoms $[19,20]$, inflammation [14], QOL, and mood disturbance [14], the increase of the number or the levels of components in peripheral blood [21], and the increase of the 5-year survival rate in cancer patients [17]. The other three RCTs showed no significant differences in outcome measures between the intervention group and the control group [15, 16, 18]. Almost all of the included CCTs, except one [24], showed a favorable effect of qigong exercise on health outcomes in cancer care.

There is a great variability in outcome measures in the studies examined in this review. Specifically, 14 studies have examined the effects of qigong on physical and psychosocial outcomes, and 15 studies have examined the effects of qigong on biomedical outcomes among cancer patients. Among physical and psychosocial outcomes, improvement of symptoms is the most frequently used outcome measure in the included studies, followed by psychological distress, QOL, survival rate, and fatigue. Other outcomes less commonly employed in these studies include physical functioning, response rate, tumor size, and body weight. Due to the variability of the results of the included studies and a high risk of bias in most of the included studies which may have led to false-positive results, it is difficult to draw a conclusion about the efficacy and the effectiveness of qigong exercise on physical and psychosocial outcomes in supportive care to cancer patients. Two outcomes that may see benefit are symptoms (including fatigue) and QOL, even though a few studies on QOL $[15,16]$ and symptoms $[15,18]$ have not demonstrated a desirable effect of qigong exercise in cancer care, possibly due to very limited sample size in the studies. With regard to the studies on biomedical outcomes examined in this review, it seems that there is a consistent tendency whereby the patients treated with qigong therapy in combination with conventional methods enjoyed significant improvements in immune function than the patients treated with conventional methods alone.

A careful examination of the included studies reveals some methodological flaws that exist in current studies on qigong intervention. The first limitation is sample size. Of the 23 included studies, seven studies were based on a total sample of less than $50[15,18,19,23,25,27,30]$ with the smallest number being 20 participants. Nine studies were conducted with a total sample of more than 50 but less than $100[16,20-22,29,31-33]$. Small sample size in these studies might lead to a type II error for their results. Moreover, sample size in nearly all of the included studies was not justified except one study [16] in which the calculation of sample was presented.

Second, the intervention designs in the included studies are problematic. In nearly all of the included prospective studies, qigong exercise was preferentially provided to the intervention groups as an additional group therapeutic modality to the usual medical care or chemotherapy that was applied to both intervention group and control group, whereas the control groups did not have a matched number of social contact hours with co-participants. Thus, these studies might be subject to potential risk of performance bias, and a placebo effect might have occurred in patients who enjoyed participation in group activities and the contact with other persons. This design limitation needs to be addressed in future studies.

Heterogeneity in the quality and the dosage of qigong intervention across the included studies is another problem. Of the 23 included studies, the style of qigong exercise was reported only in nine studies [14-16, 21-23, 27, 29, 30], which varied greatly. Practically, many styles or forms of qigong exercise, e.g., Guo-Lin Qigong, Chinese Taiji FiveElement Qigong, and Chan-Chuang Qigong, have been developed. Basically, qigong exercise can be divided into different types including meditative qigong (quiescent qigong known as jing gong in Chinese), dynamic qigong (active qigong known as dong gong in Chinese), and a combination of the two (jing dong gong). The intensity of exercise is often different for these types of qigong exercise. In some studies, a term "medical qigong" was used and was defined as "the prescription of qigong recommended or designed by a medical practitioner to improve the health of patient" $[14,15]$. According to this definition, the form and the intensity of exercise are not standardized yet. Moreover, the frequency of qigong exercise was reported only in less than half of the studies examined in this review, with a great disparity. Therefore, there is a great variability of the dosage and quality of qigong exercise across the included studies, which also make it difficult to compare and synthesize the results of these studies. It will be better to transform the intensity of qigong exercise to metabolic equivalents (METs), which is a scientific and standardized measure of the intensity of physical exercise [37], in future studies so as to increase the comparability of the results across different studies.

Lastly, a high risk of bias exists in most of the included studies. Of the 23 included studies, the methods of randomization were described in only three studies [14-16], details about allocation concealment were presented in only one study [16], and the proportion of drop-outs and withdrawals were reported in only three studies [14-16]. Other studies described neither their methods of sequence generation or allocation concealment nor the proportion of drop-outs or withdrawals, and the evidence was thus weakened for the results of these studies. The fifteen included CCTs, most of them were poor-quality cohort or case-control studies, were subject to high risk of selection bias, making that the synthesis and generalization of the results should be treated with caution. 
Previous reviews have suggested three possible mechanisms (improvement of immune functions, improvement of microcirculation, and increase in pain threshold) of the effects of qigong exercise for cancer patients $[6,7]$. However, the findings of the current review suggest that there is a lack of evidence to date to support the postulations that qigong exercise can raise the pain threshold in patients with cancer. Although most of the included studies on biomedical outcomes suggest that qigong exercise may lead to an improvement in immune function among cancer patients, but it is still unclear whether the improvement of immune function is sufficient to provide definitive benefits to the patients, since most of the included studies focus on antibody response or the enumerative measures of immunity in peripheral blood, and the studies on functional measures or cellmediated cancer-specific immunity, which is the key component of immunity for cancer cells, are very limited. Given the heterogeneity in outcome measures and the limited number of RCTs in the field, better designed studies and scientific evidences are needed to test the statement that qigong therapy may improve cancer patients' immune functions.

Overall, study quality was poor for most of the studies examined in this review. Of the 23 included studies, only three studies could be ranked " $A$ " in the levels of evidence; eleven studies were ranked " $\mathrm{B}$ " and nine studies were ranked "C". Jadad scale scores for the eight included RCTs ranged from 1 to 3 , with a value of 1 for five RCTs. Therefore, studies with higher quality are warranted in the future.

Several limitations may exist in the current review. As with most systematic reviews, there is potential incompleteness of the evidence reviewed. Another limitation may be related to selective publishing and reporting in the literature, which is also a major cause of bias. In addition, we were unable to perform meta-analyses due to the limited number of RCTs and heterogeneity of outcome measures in the included studies.

\section{Conclusions}

Due to limited number of RCTs in the field and methodological problems and high risk of bias in the included studies, it is still too early to reach a conclusion about the efficacy and the effectiveness of qigong exercise as a form of health practice adopted by the cancer patients during their curative, palliative, and rehabilitative phases of the cancer journey. Since qigong exercise is preferred and widely practiced among patients with various cancers in China and Asia, further well designed large-scale placebo-controlled, randomized studies with validated outcome measures are particularly needed.
Acknowledgements We would like to thank the Hospital Authority of Hong Kong for the support of this systematic review.

Conflict of interest The authors declare no conflict of interest. The authors have had full access to all the data in the study and thereby accept full responsibility for the integrity of the data and the accuracy of the data analysis.

Role of funding source This review was supported by the Hospital Authority of Hong Kong. The funding sources had no further role in the design; data collection, and analysis of this work.

Open Access This article is distributed under the terms of the Creative Commons Attribution Noncommercial License which permits any noncommercial use, distribution, and reproduction in any medium, provided the original author(s) and source are credited.

\section{References}

1. Fouladbakhsh JM, Stommel M (2010) Gender, symptom experience, and use of complementary and alternative medicine practices among cancer survivors in the U.S. cancer population. Oncol Nurs Forum 37(1):E7-E15

2. Goldstein MS, Brown ER, Ballard-Barbash R, Morgenstern H, Bastani R, Lee J et al (2005) The use of complementary and alternative medicine among california adults with and without cancer. Evid Based Complement Alternat Med 2(4):557-565

3. Molassiotis A, Scott JA, Kearney N, Pud D, Magri M, Selvekerova S, Bruyns I et al (2006) Complementary and alternative medicine use in breast cancer patients in Europe. Support Care Cancer 14 (3):260-267

4. Deng G, Cassileth BR, Yeung KS (2004) Complementary therapies for cancer-related symptoms. J Support Oncol 2(5):419-426, discussion $427-419$

5. Tang JL, Liu BY, Ma KW (2008) Traditional Chinese medicine. Lancet 372:1938-1940

6. Chen K, Yeung R (2002) Exploratory studies of qigong therapy for cancer in China. Integr Cancer Ther 1(4):345-370

7. Chen K, Yeung R (2002) A review of qigong therapy for cancer treatment. J Int Soc Life Inf Sci (ISLIS) 20(2):532

8. Lee MS, Chen KW, Edzard E (2010) Supportive cancer care with qigong. In: Cho WCS (ed) Supportive cancer care with Chinese medicine. Springer, New York, pp 77-94

9. Lee MS, Chen KW, Sancier KM, Ernst E (2007) Qigong for cancer treatment: a systematic review of controlled clinical trials. Acta Oncol 46(6):717-722

10. Oxford Centre for Evidence-based Medicine. Levels of Evidence (March 2009). Available at: http://www.cebm.net/index.aspx? $\mathrm{O}=1025$. Accessed 10 Nov 2010

11. Jadad AR, Moore RA, Carroll D, Jenkinson C, Reynolds DJ, Gavaghan DJ et al (1996) Assessing the quality of reports of randomized clinical trials: is blinding necessary? Control Clin Trials 17(1):1-12

12. Jüni P, Altman DG, Egger M (2001) Systematic reviews in health care: assessing the quality of controlled clinical trials. BMJ 323 (7303):42-46

13. Higgins JPT, Green S (2008) Cochrane handbook for systematic reviews of interventions. Wiley-Blackwell, Chichester

14. Oh B, Butow P, Mullan B, Clarke S, Beale P, Pavlakis N, Kothe E, Lam L, Rosenthal D (2010) Impact of medical qigong on quality of life, fatigue, mood and inflammation in cancer patients: a randomized controlled trial. Ann Oncol 21(3):608-614 
15. Oh B, Butow P, Mullan B, Clarke S (2008) Medical qigong for cancer patients: pilot study of impact on quality of life, side effects of treatment and inflammation. Am J Chin Med 36(3):459-472

16. Lam SWY (2004) A randomized, controlled trial of Guolin qigong in patients receiving tanscatheter arterial chemoembolisation for unresectable hepatocellular carcinoma. Master's thesis. Hong Kong: University of Hong Kong

17. Fu JZ, Fu SL, Qin JT (1996) Effect of qigong and anticancer body build herbs on the prognosis of postoperative patients with cardiac adenocarcinoma. 3rd World Conf Acad Exch Med Qigong, Beijing, China

18. Fu JZ, Zou ZK (1995) Qigong combined with chemotherapy in the treatment of gastric cancer. In: Lin ZP (ed) Understanding of true qi cultivation and sublimation. Chinese Publisher of Constructive Materials, Beijing, pp 157-158

19. Fu JZ, Wang SM (1995) Qigong plus herbal medicine in treating late-stage stomach cancer in the elderly. In: Lin ZP (ed) Understanding of true qi cultivation and sublimation. Chinese Publisher of ConstructiveMaterials, Beijing, pp 155-157

20. Wang CH, Wang BR, Shao MY, Li ZQ (1993) Clinical study of the routine treatment of cancer coordinated by qigong. 2nd World Conf Acad Exch Med Qigong, Beijing, China

21. Luo S, Tong T (1988) Effects of vital gate qigong on malignant tumor. 1st World Conf Acad Exch Med Qigong, Beijing, China

22. Lee TI, Chen HH, Yeh ML (2006) Effects of chan-chuang qigong on improving symptom and psychological distress in chemotherapy patients. Am J Chin Med 34:37-46

23. Hong EY (2003) The effect of Yudongkong exercise in fatigue, difficulty of daily activities and symptoms of side effect in advanced gastric cancer patients receiving chemotherapy. $\mathrm{PhD}$ dissertion. Seoul, Korea: Yonsei University

24. Wang Y, Ye M (2002) Analysis of psychological error factors in assessment of therapeutic effect of qigong rehabilitation in cancer patients. Acta Univ Trad Med Sinen Pharm Shanghai 16:20-22

25. Fu JZ (1993) Treatment of advanced gastric cancer in the aged by the combination of qigong and medical herbs. 2nd World Conf Acad Exch Med Qigong, Beijing, China
26. Zheng RR (1990) Observation of 100 cases with comprehensive qigong therapy for treating later-stage cancer. World Qigong 3:19

27. Peng ZS, Chen XY, Wen YZ, Ni YW, Deng GY (1989) Clinical observation of 20 patients with malignant gastrointestinal tumors treated by supplementary Qigong. 2nd Int Conf on Qigong, Xi'an, China

28. Sun QZ, Zhao L (1988) Clinical observation of qigong as a therapeutic aid for advanced cancer patients. 1st World Conf Acad Exch Med Qigong, Beijing, China

29. Yeh ML, Lee TI, Chen HH, Chao TY (2006) The influences of Chan-Chuang qi-gong therapy on complete blood cell counts in breast cancer patients treated with chemotherapy. Cancer Nurs 29 (2):149-155

30. Yu H (1996) The effect of Qigong exercise on anti-oxidant capacity among cancer patients. Chin J Sports Med 03:233

31. Ye M, Wang Y, Mao CX, Zhang RH (1992) The effect of qigong exercise on unscheduled DNA synthesis of the peripheral blood lymphocytes of cancer patients. 4th International Symposium on Qigong, Shanghai, China

32. Lo J, He C, Lu H, Wang L (1990) Changes of peripheral blood cell population and immune functions in 31 nasopharyngeal carcinoma (NPC) patients treated with radiotherapy and qigong. 3rd Nat Acad Conf on Qigong Science, Guangzhou, China

33. Qi YQ (1989) Changes of lymphocytes lipase in cancer patients treated with Qigong and Chinese medicine. J Nanjing Univ Trad Chin Med 01:40

34. Xu H, Xue H, Zhang C, Zhou Q, Shao X (1990) Clinical study of the effect of qigong practice on the Cu-Zn SOD activity in cancer patients. 3rd Nat Acad Conf on Qigong Science, Guangzhou, China

35. Xu HF, Qi YQ (1989) Preliminary study on qigong and immunity. 2nd Int Conf on Qigong, Xi'an, China

36. Xu HF, Wang GM, Xue HH, Zhanbg CM, Wang JM, Qi YQ (1988) Observation of T lymphocytes by ANAE staining in the clinical application of qigong. 1st World Conf Acad Exch Med Qigong, Beijing, China

37. Jette M, Sidney K, Blumchen G (1990) Metabolic equivalents (METS) in exercise testing, exercise prescription, and evaluation of functional capacity. Clin Cardiol 13(8):555-565 